\title{
When humans using the IT artifact becomes IT using the human artifact
}

\author{
Dionysios S. Demetis \\ Hull University Business School \\ d.demetis@hull.ac.uk
}

\begin{abstract}
Following Lee \& Demetis [20] who showed how systems theorizing can be conducted on the basis of a few systems principles, in this paper, we apply these principles to theorize about the systemic character of technology and investigate the role-reversal in the relationship between humans and technology. By applying systems-theoretical requirements outlined by Lee \& Demetis, we examine conditions for the systemic character of technology and, based on our theoretical discussion, we argue that humans can now be considered artifacts shaped and used by the (system of) technology rather than vice versa. We argue that the role-reversal has considerable implications for the field of information systems that has thus far focused only on the use of the IT artifact by humans. We illustrate these ideas with empirical material from a well known case from the financial markets: the collapse ("Flash Crash") of the Dow Jones Industrial Average.
\end{abstract}

\section{Introduction}

The field of Information Systems (IS) rests largely on examining the contextual use of technology within social (sub)-systems and organizations. In such a context, the relationship between the social and the technical has always been of special interest to IS researchers. Ultimately, this interest is applied to the interactions between humans and information technology, and at the center of attention, one can often find the concept of the IT artifact. From considering the IT artifact as an ensemble of hardware and software [27] to bundles of material and cultural properties that are recognizable and emerge from ongoing socioeconomic practices [34], or even to sociotechnical assemblages [38], one thing is clear: the concept of the IT artifact has changed substantially over the years. In fact, the ontological dimensions upon which the "IT artifact" has come to be considered have shifted so much that Steven Alter's suggestion was to "retire" that concept altogether from the lexicon of IS scholarly debate as it has outlived its usefulness [1]. While this is not an

\author{
Allen S. Lee \\ Virginia Commonwealth University \\ allenslee@alum.mit.edu
}

essay about the concept of the IT artifact per se, we do make the argument that the nebulous character of that concept is due to a much larger (though subtle) phenomenon at play: the transition of technology from artifact to system. In fact, the contextual richness that has been added to the concept of the "IT artifact" after its first use by March and Smith [27], can be re-interpreted as a recognition of such a transition. Inspired by the post-humanist tradition that reflects on the boundaries between humans and technology, we take a different approach and develop a systems theoretical description of the transition from artifact to system. We argue that people are becoming agents of the (system of) technology.

Over time, technology has penetrated society to such a degree that even basic functions now seem almost inconceivable without technology. Indeed, the level of dependence of society on technology has become so deep that - in a large number of fields there are now no manual fallback plans in cases of technological failure. By and large, even when technology fails, we tend to rely on more technology for rectifying the problems of technological use. Also, the rising trend of technologized decision making that has taken certain fields by storm is even more alarming. In the foreign exchange markets for example, 85 percent of all trading is conducted by algorithms alone, i.e., without any human intervention; this led the scholars that investigated the phenomenon to call this the "Rise of the Machines" [10]. In the UK, the "ultra-high-speed version of algorithmic trading, high frequency trading, is estimated to account for over $77 \%$ of transactions in the UK market" [40, p.5].

A skeptic of our position who might seek to argue against the trend of technologized decision making, might pose this question: Is it not the case that the designers of algorithms are humans? And if so, then couldn't someone consider the role of algorithms (and of technology at large) as an extended application of human decisions?

Our challenge is to convince the reader to the contrary. For this purpose, we render this issue through a few intertwined questions: How does technology subvert and subdue human decisions? 
What characteristics can be identified (with the help of systems theorizing) for this new role that technology has assumed and how does this constitute the emergence of a system of technology? Even more crucially, how is it that humans become "artifacts" being shaped and used by technology in this seemingly counterintuitive - role reversal?

As we will see through our example of the Flash Crash regarding the Dow Jones Industrial Average, the role of technology leads us to consider a seemingly radical idea at first - but one that we believe is an accurate reflection of how technology shapes social systems and subjects humans to forces that cast them out to the environment, outside of what has become technologized decision making; instead of the IT artifact being shaped and used by humans, humans can actually be considered as "artifacts" being shaped and used by machines. In this view, it is humans that must react to technological stimuli rather than technology that must react to human stimuli where, furthermore, the technological stimuli are emergent and not pre-designed (or pre-programmed) in any way. This also assumes that while the controllability of technology can be achieved at a micro-scale (where one could assert that the link between designers and - control of - artifacts is strict), at a macro-scale, technology exhibits emergent non-linear phenomena that render human controllability irrelevant $[4,14]$. This creates a significant circularity wherein the systemic role of technology demands a higher degree of autonomy to be granted in the design of technological artifacts, where this then leads to a deeper systemic function of technology that leads to more demands for autonomy. Humans increasingly find themselves in the environment, outside of these dynamics.

\section{Examples}

Indeed, one can find a wealth of examples where technological autonomy has developed into a system that takes over important decisions - and humans find themselves outside, i.e., cast out to the environment, outside of these decisions. In such examples, human agency - "acting on behalf of another, or providing a particular service" [16] is being replaced by technologized agency. In categorizing the World Wide Web for instance, Yahoo (to bring up one example) uses proprietary algorithmic robots (known as bots) to create a searchable database that then ranks users' search results based on their search queries. The structuring of the bot-generated entries contains the logic of how something will be "made searchable," though due to the complexity of the task, the interaction between bots and websites that are indexed must be unsupervised. But while the millions of pre-indexed search results give the illusion of choice, almost $90 \%$ of humans don't get past the top ten [18]. The whole process feels like a "search on the Internet" but it is actually a restricted human search of a technological pre-search of the Internet: the "search of a search" that constitutes a $2^{\text {nd }}$ order phenomenon. Hence, this is a case of a human reacting to technological stimuli (i.e., an individual person reacting to the algorithmically generated search results intended to steer the person's behavior) rather than a technology reacting to human stimuli (i.e, a neutral search algorithm providing objective results to best serve a human using the technology).

Another well-known example comes from Amazon. The vast majority of prices are defined by algorithms in so far as Amazon vendors "use algorithmic pricing to ensure that they can automatically change their product prices based on a competitor" [39], with the result that vendors are being forced to engage in this practice for fear of losing out to the competition. Meanwhile, the algorithmic interactions between vendors carry the possibility of developing unpredictable consequences. Such algorithmic pricing on Amazon can be found in the example of the book entitled The Making of a Fly by evolutionary biologist Peter Lawrence. This book came to be priced at $\$ 23,698,655.93$ (plus \$3.99 shipping) as two sellers were using algorithms to adjust the price of the book in response to one another. It took 10 days for humans to notice and intervene to bring back the prices to normal levels [43]; ironically, "normal levels" merely indicated a temporary human decision that would allow the continuation of algorithmic pricing.

Similar examples where human decisions and human reactions find themselves outside the boundary of technologized decision making can come from any domain. In law for instance, legal analysts are being replaced in complex cases by software that analyses thousands of legal documents; proprietary e-discovery algorithms of software companies pre-structure the defense/prosecution of a case by effectively pre-selecting an extremely limited subset of documents that will then be looked at and presented in court by humans [28]. By and large, it would be fair to say that in such cases, humans become the tool through which computerized decisions are voiced. In autonomous driving, Google's self-driving car assumes full control of the vehicle, and while in principle, it might appear as if human decisions can always override the technologized driving process, there are no strict 
criteria which humans could apply in such a process (as the decisions of the vehicle cannot but depend on the unpredictable circumstances that emerge in its environment); the self-driving car caused its first crash when the human user was not certain whether he should have intervened [13]. In news, Bloomberg now uses automated sentiment analysis to provide financial news about companies in an automated way; this can further assist its customers in making financial decisions, "without having to consume the content" [7]. In the job market, many of the world's biggest companies use automated vetting software to screen CVs for particular job applications without even glancing at the CVs on the first round [30]. Even in the judiciary, the discussion on whether computers would be better and fairer judges has started since 1977 and has considered the algorithmic imposition of verdicts and fines [12].

The next and third section of this essay provides a review of a selection of the general requirements proposed by Lee \& Demetis [20] for systems theorizing, for the purpose of theorizing about technology itself as a system in the systems theoretical sense. This section also serves to pose reflective questions about the deep interference of technology in society and highlight elements that ought to be considered for technology in this context.

The fourth section will present the case of the Flash Crash in the Dow Jones Industrial Average Index and will review the key characteristics of that case by considering how technology shaped the minicrisis through automated execution strategies.

In the fifth - and final - section of the essay, we shall conclude with a brief discussion and reflect on the consequences of the basic systemic principles of technology.

\section{Theoretical conditions for technology itself as a system}

In exploring a set of requirements/principles that systems theorizing needs to satisfy, Lee \& Demetis [20] consider general principles that are based on the founders of systems theory like Ludwig von Bertalanffy [5], Kenneth Boulding [8], James Grier Miller [31] and Anatol Rapoport [36]. In their work, Lee \& Demetis [20] extract three principles from this body of systems theory and add another three from Niklas Luhmann [25]; they maintain that systems theory is particularly relevant for IS research and a rich theory to draw from (regardless of any epistemological differences amongst different scholars). In this context, we argue that systems theory lends itself in exposing the subtle emergence of technologized interferences in the relationship between humans and technology and will enable researchers (due to its abstract/generalized lexicon) to render novel cases through its concepts across several domains of application. In Table 1 below, we list the six requirements as they appear in Lee \& Demetis [20]. We give each one a code $\left(R_{i}\right)$ for when we use the requirements in this paper though it is worth stressing that we don't reflect on all six equally. Also, for the purposes of our discussion, we do not examine them in sequential order.

\begin{tabular}{|l|l|}
\hline Requirement specified in Lee \& Demetis [19] & Code \\
\hline $\begin{array}{l}\text { The whole is more than the sum of the parts } \\
\text { Goal Seeking }\end{array}$ & $\mathrm{R}_{1}$ \\
\hline $\begin{array}{l}\text { Transformation Process (of inputs into } \\
\text { outputs) }\end{array}$ & $\mathrm{R}_{2}$ \\
\hline Self-Reference \& Autopoiesis & $\mathrm{R}_{4}$ \\
\hline System/Environment distinction & $\mathrm{R}_{5}$ \\
\hline Communication & $\mathrm{R}_{6}$ \\
\hline
\end{tabular}

Table 1: Systems Requirements by Lee \& Demetis [20]

Our presentation of their requirements below is not a substitute for the original theoretical analysis rather, it will be a discussion focused on targeting those theoretical conditions for thinking about a system comprised of technology. In other words, through different systems-theoretical principles, we can focus on the following question: what systems theoretical principles would need to be adhered to, in order to consider technology itself as a system?

One of the first requirements that Lee \& Demetis [20] stipulate in $R_{1}$ is the requirement to recognize that if we consider any system as a whole then "the whole is more than the sum of the parts." Regardless of whether we take technological artifacts in one sense (e.g. a combination of hardware/software) or another (e.g. sociotechnical assemblages), this requirement still applies. Whenever any elements become interrelated and interdependent, they form a more complex system; however: "One cannot deduce from complexity alone which relations among elements are realized" [25, p.25]. Applied to technology at large, this systemic requirement demands that whatever technological "whole" is identified by an observer needs to be considered as a system that exhibits emergent properties. These properties remain irreducible to the whole's constituent elements. 
Another important requirement $\left(\mathrm{R}_{5}\right)$ is based upon the fundamental distinction between system and environment. As stressed by Lee \& Demetis [20], this requirement is absolutely critical as no system can be perceived without an environment [3]. The environment should not be perceived as some type of residual category. Instead, "relationship to the environment is constitutive in system formation" [25, p.176]. By considering this fundamental principle that constitutes a pillar of systems theory and transcends all systems theorists [17], we can pose an important question: if we consider technology as a system then what is its environment? One response would be that humans (also labeled by Niklas Luhmann as psychic systems) are in the environment outside of and around a system of technology; another would be other technologies that would not interconnect directly with a specified (by an observer) system of technology. Another option of course would be a combination of the above - an environment wherein both humans/technology reside. While all possibilities can be considered, when we describe technology as a system in itself, we make the case that - via the systemic evolution of technology and its deep penetration in society - technology as system assimilates more functions within itself; in doing so, humans are cast out to the environment around the system of technology and cease to perform the function of decision making. The function of humans in the environment outside a system of technology becomes ever more restricted in merely providing inputs through which technological decision-making can continue uninterrupted. This is of course both hard to accept (for humans) and difficult to explore. It also implies that humans simply react to what is now technologized decision making and that, by and large, human "decisions" are secondary to systems-made decisions. At the very least, humans and what they believe their own decision-making to be need to adapt to the emergence of systemic technological phenomena. Is human behavior patterning itself after, or simply being patterned by, what the system of technology generates?

One implication leading from the primacy of the system/environment distinction is that the distinction can be replicated within the system (so within any observed system, one can delineate further subsystem/environment distinctions). This is another way of saying that the system replicates that primary distinction (between system/environment) within itself. This idea of any form (defined by Luhmann as the unity of a distinction) affecting itself recursively is distilled in the concept of re-entry. Based on George Spencer-Brown [41], Luhmann makes the following remarks: "Accordingly, the re-entry of the form into the form-or of the distinction into the distinction, or of the difference between system and environment into the system - should be understood as referring to the same thing twice. The distinction re-enters the distinguished. This constitutes re-entry." $[26, \mathrm{p} .54]$

This type of re-entry is tightly connected with the concept of self-reference $\left(\mathrm{R}_{4}\right)$ : satisfying the systemic requirement for self-reference means identifying processes through which a system collects information about itself (and its own functioning), where this in turn can contribute to a change in its functioning. Through self-referential processes, certain systems (those that Luhmann calls autopoietic) continuously re-produce and maintain themselves. In this regard, considering technology as a self-referential system implies recognition of these dynamics that exhibit this form of re-entry; technology referring to technology is not a new phenomenon but again, in the context of technologized decision-making this acquires further significance. To the degree that technologized decisions become deeply embedded across different social systems, they elevate the complexity of the system of technology. This condition makes it harder for humans to gain visibility of the consequences of such systemic technologizing and, due to the excessive reliance of humans on technology, this reinforces the role-reversal between the two. The demand for further technologizing ends up with humans augmenting the systemic character of technology further.

Another important systems requirement that follows Luhmann's systems theory involves the requirement for communication $\left(\mathrm{R}_{6}\right)$. For Luhmann, who reserves the term communication to indicate a broader mechanism (instead of only an act between human individuals), communication can be considered in the following triad form: announcement/utterance (Mitteilung), information (Information), and understanding (Verstehen). Also, in the event where human beings are participating in the communication process, it would follow that the cognitive understanding (Verstehen) that would be developed by the recipient might not correspond to the intentionality of the individual conducting the utterance.

The reciprocity in communication however demands that whatever entity is receiving information following another entity's utterance will react based on its own (the former's) understanding. While we are typically used to be thinking about these processes as human-based exchanges, they can be easily applied at a more abstract level. In fact, one 
of the pioneering theoretical implications of Luhmann's systems theory was the separation of the communication process from psychic systems (i.e. humans) and its consideration as a function of society $[23,25]$. In a system comprised of technology (rather than of humans), the hardest element of the triad to consider is understanding; however, that does not have to be cognitive understanding for communication to be facilitated. In the case of a system of technology, substituting for human (or cognitive) understanding is technologized understanding; this signals the a priori acceptance or rejection of any information, which would then prompt a computational response (another utterance) on the basis of a pre-coded algorithmic rationality. This demarcates the transition from (human) reflective understanding to pre-organized understanding (that effectively collapses - or is reduced to - computerized decision making). Of course, substituting the second for the first is troubling. Also, human decisions are not just transferred across to computer decisions via the design of specific artifacts. The input from human designers is transformed $\left(\mathrm{R}_{3}\right)$ into systemtechnologized decisions/outputs through a complex nexus of technological interactions. While the goalseeking $\left(\mathrm{R}_{2}\right)$ of specific algorithms may be perceived as fixed at the micro-level, at the macro-level of the system it becomes dynamic and uncertain. The very existence of unpredictable phenomena that emerge from such technological interactions is a testament to the fact that the correspondence between inputs and outputs in this context is non-linear. As technology becomes responsible for "major systemic changes within the global financial sector... and as algorithms become ever more autonomous... we need a kind of ethical framework for developing algorithms" [44].

\section{CASE OF THE DOW JONES INDEX}

First, let us clarify that it is beyond the scope of this paper to organize and/or deconstruct all the technologically-oriented incidents behind the events of May 6, 2010 (what has become known as the "Flash Crash of $2: 45$ "). Indeed, there remain several conflicting aspects on these events $[22,32,40,44$, 46] so we will focus here only on a handful of critical aspects that illustrate how the demand for increasing computerization is framing the interactions between humans and machines. In this regard, we would also like to highlight that the complexity of this case has also been attributed to the multi-faceted role of algorithmic traders who make it almost impossible to deconstruct the case; this is acknowledged in several reports (see below).

The purpose of this section is to reflect on the case of the Flash Crash and connect some of its key aspects to a systems-based re-conceptualization of technology based on the systemic principles put forward by Lee \& Demetis [20]. This theoretical framing will allow us to consider the case of the Dow Jones index collapse as an example of technological domination-an example where the system of technology locates human artifacts in its environment, outside of and around the technology. The key sources that we will use in order to review the main findings of the Flash Crash include:

i) The Report of the Staffs of the U.S. Commodity Futures Trading Commission (CFTC) and Securities \& Exchange Commission (SEC) to the Joint Advisory Committee on Emerging Regulatory Issues.

ii) The UK report on "Crashes and high frequency trading" from the Government Office for Science (The Future of Computer Trading in Financial Markets - Foresight Driver Review - DR 7), and

iii) An analysis for Certified Public Accountants when advising investors entitled "Understanding the "Flash Crash'" that summarizes the basic characteristics of the Flash Crash in a succinct way [6].

First, in order to set the scene, it is important that we reflect on the turmoil behind the specific events in their broader context. Uncertainty in May 2010 was already widespread in the market due to the possibility of a Greek government default on sovereign debt [29]. Based on $\mathrm{R}_{5}$, we can think of this broader financial turmoil as the environmental stimulus to which the system of algorithmic traders would react. This negative market sentiment "was already affecting an increase in the price volatility of some individual securities" [9, p.1]. This set in motion the following key events before the $2: 45 \mathrm{pm}$ Crash (in chronological order):

1) A number of volatility pauses were triggered on the New York Stock Exchange (NYSE) around 1pm; individual equities began to increase above average levels.

2) The S\&P 500 volatility index rose by $22.5 \%$ by $2: 30 \mathrm{pm}$. In conditions of such volatility, investors moved their capital away from investments that were considered to be highrisk and towards safer options while the Dow Jones Industrial Average was down by $2.5 \%$ (due to selling pressure). 
3) Buy-side liquidity in the "E-Mini" and the "SPY" (the E-mini S\&P 500 futures contracts and the S\&P 500 SPDR exchange traded fund) had suffered $55 \%$ and $20 \%$ declines respectively.

4) Then, more critically, at about $2: 32 \mathrm{pm}$, a large fundamental trader (a mutual fund complex) "initiated a sell program to sell a total of 75,000 E-mini contracts (valued at approximately $\$ 4.1$ billion) as a hedge to an existing equity position" $[9, \mathrm{p} .2]$.

The last step in the event is viewed as one of the critical triggers that led to the Flash Crash. But before we develop that further, we need to make an important observation in this context. Whereas traders can choose how much human judgment is involved when executing a trade (e.g. the trader can choose to enter orders manually in different time intervals, or indeed, outsource the process to a thirdparty that will manage this process by conducting block-trades), over the years, the interference of human judgment came to be perceived (by humans themselves) as an obstacle. For example, Lewis [21] remarks that during the crash of 1987 when the US stock market fell sharply by $22.61 \%$, "some Wall Street brokers, to avoid the orders their customers wanted to place to sell stocks, simply declined to pick up their phones ... this time the authorities responded by changing the rules - making it easier for computers to do the jobs done by those imperfect people. The 1987 stock market crash set in motion a process - weak at first, stronger over the years - that has ended with computers entirely replacing the people" [21, p.3]. The need for computerization was paired with the inexorable need for speed; the intensification of these dynamics led to Wall Street's "speed war." An example of that saw the development of a superfast fiber-cable route between Chicago and New York by Daniel Spivey, just to shave "3 milliseconds off of the previous route of lowest latency" [42]. Speed matters; the fastest algorithm can exploit a large volume of minor discrepancies between markets and this is like "picking gold coins from the floor" [42].

In such a context where 825 miles of fiber are laid down through mountains, tunnels, and rivers, in the straightest line possible in order to shave off 3 milliseconds of trading, one can begin to contemplate how human beings are perceived (by the designers of algorithmic trading systems) where the average reaction time (for the click of a button) is 215 milliseconds. Human beings are not just slow - they are (almost) redundant in such a market (though - as we shall see - they still serve the purpose of recalibrating (parts of) the technological system that is actually making the decisions). More recently, the search for more speed that would allow even faster versions of algorithmic trading to take place has led to large investments in microwave communications with the goal of shaving off an extra 1 millisecond in transacting [45].

It was in this context that the large fundamental trader initiated a sell program for the $\$ 4.1$ bn trade; this was executed automatically and thus an algorithm took the task to sell $\$ 4.1 \mathrm{bn}$. In this case, the trader "chose to execute this sell program via a ... 'Sell Algorithm' that was programmed to feed orders ... but without regard to price or time" [9, pp.2-3]. The algorithm only took volume into consideration. But while the initial problems were indeed created by the algorithm of the fundamental trader, they were then "amplified by the strategic behavior of the HFT [High-Frequency Trading]" [40, p.11]. HFT is yet another name for algorithmic trading or black-box trading. The consequences of the amplification of the Sell Algorithm by yet other algorithms created a dynamic exchange between technologies of the same type. Based on $\mathrm{R}_{3}$ this can be framed in the following general form: Technology $A$ provides a stimulus for exciting technology $B$, and the output of B's operation(s), as feedback and input to $A$, recursively shape the environment of technological subsystem $A$.

One can conceptualize this situation through systems theorizing by means of considering alternative observing perspectives with which to explore the system/environment distinction $\left(\mathrm{R}_{5}\right)$. First, if the Sell Algorithm executed by the large fundamental trader is considered from the perspective of technological subsystem A, then in its environment one can observe: a) the totality of all other automated execution algorithms (let's call those $A \operatorname{lgo}(1)$, $A \operatorname{lgo}(2), \ldots A \operatorname{Algo}(n))$, and $\mathrm{b})$ the transaction outcomes from human traders (though as we saw in the introduction, the volume of their transactions is becoming more and more limited). Similarly, if we take the different observing perspective of another HFT, say Algo(2), which we define as our system, we would observe the Sell Algorithm in its environment, along with all other automated execution algorithms [Algo(1), Algo(3), ...Algo(n)] and the human traders. Naturally, for fair competition reasons, the trading logic of any given $\operatorname{Algo}(n)$ is hidden from all other algorithms. Considering both of the mentioned observing perspectives in tandem at a meta-level (one that would be applicable for any automated execution), we have a system that is stimulated by the reactions it itself triggers upon its own environment.

Indeed, while the sell pressure established by the "Sell Algorithm" was initially absorbed by High- 
Frequency Traders (HFT's), - at about 2:44pm HFT's started to sell contracts aggressively. Then, the "Sell Algorithm" used by the large trader responded to the increased volume by increasing the rate at which it was feeding the orders into the market, even though orders that it already sent to the market were arguably not yet fully absorbed" [9, p.3]. This type of self-reference $\left(\mathrm{R}_{4}\right)$ can be portrayed as a "negative spiraling effect ... (where)... HFT may have a destabilizing effect through its endogenous self-excitation nature within the (small) pool of participants" [40, p.11]. This unsupervised self-excitation of technology at the level of the financial market is based upon a coupling between technology and technology, a condition that relegates humans to the external environment of that system. This has both significant and specific implications.

In the events that unfolded, the Dow Jones Industrial Average plunged 998.5 points. This became known as the Flash Crash of 2:45 (and while the index recovered some moments later, it wiped out value from several companies). After five months of investigations, the U.S. Securities and Exchange Commission (SEC), along with the Commodity Futures Trading Commission (CFTC), attributed the decline to the automated execution of orders despite the fact that no specific reason/trigger/algorithm could be identified for the event. In fact, "the exact reason or reasons for the so-called flash crash remain obscured by the mechanics of the electronic trading systems that execute millions of buy-and-sell orders during the course of a single trading day. Some initially blamed the crash on a 'fat finger,' (meaning a big individual investor) while others contended that essentially unregulated electronic trading platforms were the culprit. Others even questioned whether terrorists or hackers were behind the dramatic drop" [6, pp.40-41].

The difficulty in identifying a specific cause renders the very idea of cause-and-effect (itself a nonsystematic idea) problematic in this context; this constitutes an irony, considering that the repeatable operations of technology usually allow the identification/back-tracking of effects. Of course, without recursive feedback, technology as a system would not exhibit such ramifications in the first place. In this regard, what would otherwise be conceptualized as cause and effect is better conceptualized as a web of back-and-forth impacts distributed amongst the complex interactions within the broader system of technology. There is no "error" in the individual technologies themselves (i.e. the algorithms); no bug needs be rectified. Thus, nonattribution of error to a single algorithm leads us to consider this as a systemic phenomenon that emerges out of the complex interaction of multiple automated execution technologies $\left(\mathrm{R}_{1}\right)$.

Of course, the flash crash would have been impossible at such a level without the complex interactions between algorithms. As the testimony of SEC Chairman Mary Schapiro confirmed to the US Congress: "automated trading systems will follow their coded logic regardless of outcome, while human involvement likely would have prevented these orders from executing at absurd prices" [37, p.7]. The unpredictability with which automated algorithms feed off each other creates emergent conditions that can destabilize any system that technology itself penetrates. For the financial system that serves such an important function within the broader system of society, the implications are clear: Market outcomes are guided by computerized decisions that are executed algorithmically. While individual algorithms may reflect the general intentions of their designers, the algorithms as a whole find themselves within a far more complex environment (that they themselves help to constitute and create). In such conditions, algorithms feed off each other and within the demands for millisecond transacting and communication $\left(\mathrm{R}_{6}\right)$ - create another version of the financial system where transacting decisions are executed in a technologized fashion; these lead to unpredictable consequences. Ultimately, this rearranges the roles of technology and humans.

For example, while the chaotic behavior was continuing in the flash crash, another algorithm was triggered, known as the Stop Logic Functionality of the Chicago Mercantile Exchange (CME). This was automatically executed in order to prevent any further price movements over an excessive range. By effectively pausing the market momentarily, this gives time to humans to recalibrate any parameters and re-launch the algorithms in the pit of algorithmic trading. The need to include Stop and Pause algorithms in order to contain any emergent uncertainties from the automated behavior of other buy/sell algorithms illustrates two things: first, how the "controllability" of technology is dependent on more technology, and, b) how restricted the role of human beings has become in the actual decisionmaking process in the financial system. To a large degree, "People no longer are responsible for what happens in the market, because computers make all the decisions" [21, p.270]. Whatever intentionality is imbued within specific algorithms by its designers, this dissipates quickly. Humans are merely there to "recalibrate" the re-launch of algorithms so that the latter can be pitted against each other in millions of millisecond-transactions, the net result of which is unpredictable. 
Of course, once the demand for algorithmic decisions is set firmly, further complications can be considered. For example, new "crash algorithms will likely be developed to trade during periods of market stresses in order to profit from these periods" [40, p.4]. Algorithms will also be developed that will attempt to exploit to the maximum the number of times they can offer/request something from the market. The patterns of some of these algorithms have been discovered on a few occasions, and what is rather astonishing is the speed with which orders may be placed. Below is a visualization of an algorithm (labeled the "Knife" by the company Nanex) where the algorithm transacts around 7 times every 60 milliseconds; in the image below, the dotted vertical lines indicate the $60 \mathrm{~ms}$ intervals while the whole duration represented in the image below is 1.6 seconds. The analysis of the company on highfrequency trading found "cases where one exchange was sending an extremely high number of quotes for one stock in a single second: as high as 5,000 quotes in 1 second!" That's 5 quotes (for the same stock) every millisecond. As the company states: "Even more disturbing, there doesn't seem to be any economic justification for this" [33].

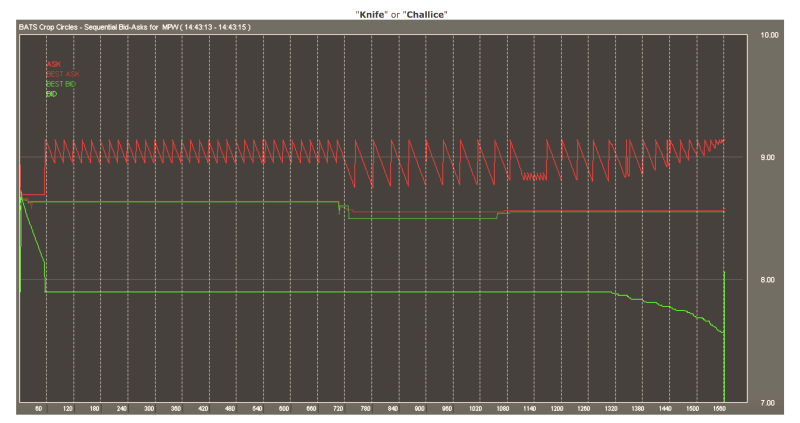

Figure 1: Visualization of algorithmic formations during trading (with kind permission from nanex.net)

Also, one can consider here algorithms that are designed to find the digital footprints of each other. By considering such algorithms that collaborate in specific market conditions, we could see the emergence of unfair algorithmic competition; and indeed, with the increasing attention that has been given to information security and cybersecurity breaches [15], automated financial trading may become subject to hackers or terrorists that will seek to create financial instability deliberately (e.g. by deactivating/modifying a stop algorithm). Whatever the destabilization effect, the speed of such transacting and the practical alienation of the human factor, relegated to the environment of the system of technology, can lead to an algorithmic war. To summarize, this involves algorithms that structure the market (by automated execution), anti-systemic algorithms that could destabilize the system (e.g. by creating complexity in automated transacting and generating a financial crisis or other destabilization phenomena), crash algorithms that may exploit financial instability for profit, as well as the algorithmic response of the regulators to the crisis (by stop/pause algorithms). Due to the intrinsic complexity and the multiple entities involved in these cases, no single entity (human or algorithm) can monitor all financial interactions taking place at any given point in time; this realizes the necessary conditions for a system that maintains and sustains itself via the function of automation: the system of technology.

\section{Discussion: from Artifact to System}

Despite our use of the Flash Crash incident, we are not making a value judgment whether algorithmic trading is good or a bad. Indeed, there are scholars arguing that - overall - high-frequency trading by algorithms may even be beneficial for market liquidity $[2,11,19]$. However, we would like to draw the attention of our readers to the fundamental imbalance in human/computer decision-making and highlight the interest that this poses for the field of IS. At a minimum, the conditions described above should allow us to reflect further on the transition from IT as an artifact (a tool shaped and used by humans to serve human ends) to IT as its own system (which in turn regards humans as tools for it to shape and use to suit the IT system's own ends). In that condition, technology expresses itself through emergent phenomena and cannot be controlled in a causal way. Of course, this runs contrary to the design of technologies with a specified coded rationality.

The logic with which any given technological artifact interacts with other technological artifacts also requires reconsideration. In circumstances like those we have described in the previous section for the flash crash, one cannot deny that there is a high degree of complexity (not even a prolonged investigation could identify the "causes" as these are distributed and not linked to single entities). But there is another reading of complexity that could illuminate an additional aspect. Luhmann defines complexity "as a measure of the incapacity of a system to relate each element to every other one, be it in the system itself (system complexity) or in its environment (environmental complexity). ... Complexity means the necessity of selective relations and, since relations specify what elements are possible within the system, complexity also means contingent 
elements. The analysis of complexity leads back to the notion of self-referential, self-organizing systems" [24, p.993]. Applied to the conditions that this view of complexity poses for technological interconnections, we can infer the following: different technological artifacts must succumb to a restriction of their individual coded rationalities as a precondition to interconnect. A very simple example of this is the following (this does not however capture the technologized complexity of more complex networks or entire function systems of society like the economic system): Suppose we have two different companies ( $\mathrm{A}$ and $\mathrm{B}$ ) that engage in algorithmic trading and both companies are the only two companies that want to sell/buy stock to/from another company $\mathrm{X}$. We assume that A will not know what B's strategy would be and vice versa. By participating in algorithmic trading, A wants to sell the stock of company $\mathrm{X}$ and has set an algorithmic parameter between $\$ 20$ and $\$ 10$ (we're making the hypothesis here that price is the only criterion). Company $\mathrm{B}$ on the other hand has set an algorithmic parameter to buy the stock between $\$ 6$ and $\$ 12$. Both algorithms are executed so that their strategies are optimized (so the algorithm would first start exploring the sale of the stock at $\$ 20$ before going down to $\$ 19.5, \$ 19$, and so on - one can inject here several other conditions like the time-frequency with which the drop would take place, the value of the drop in each step (say $\$ 0.10, \$ 0.50, \$ 1$ ), etc). Similarly for B (starting from \$6). But before even this process starts to take place for $\mathrm{A}$ and $\mathrm{B}$, the subset of pre-coded rationalities, specifically the subset within the range between $\$ 12-\$ 20$ for $\mathrm{A}$ and \$6-\$10 for $\mathrm{B}$, constitutes a non-existent set for an observer who would have visibility of both. The establishment of allowed relations between A and B as a prerequisite for inter-connecting, necessitates a restriction from the full spectrum of available possibilities for each one. Even in this simple example, seemingly well-defined thresholds that express pre-coded rationalities are facing restrictions based on their environment. The extent of unavoidable restrictions in element-interconnection cannot be anticipated by the designers of the original systems of A and B. Despite the strict controls that may be imposed by designers, an ensemble of artifacts can evolve additional systemic conditions.

Then, if we consider more realistic assumptions like: a) dynamic ranges in price (say between $\$ \mathrm{x}$ and $\$ y$ ), which will not be fixed, or b) algorithms that take input from their (uncertain and dynamic) environments in order to "determine" that (temporary \$ $\mathrm{x}-\$ \mathrm{y}$ ) range, we can see how millions of transactions and millisecond-timeframes fuel systemic complexity. What is the role of designers in this case? What meaning does "controllability" acquire in the context of an "artifact?" Hence, the design of any technological artifact (such as an algorithm) with a specific coded rationality is simply the starting point through which that artifact will be allowed to partake in the complex nexus of algorithmic exchanges. Through those, all technologized trading algorithms "design" the market collectively and create an asymmetry between humans/technology; in those domains where technology has become more dominant in overtaking human decision-making, this implies a severe restriction of human agency, intentionality, participation, and decision-making.

This shift that we describe does not only imply that "technologies create the ways in which people perceive reality" [35, p.21]. In taking decision making away from humans, technologized decision making within the context of a system of technology creates a reality that casts humans out to its environment. Alas, human decision-making is becoming more and more restricted in a support/"tool-like" role that allows for the continuation of complex and invisible (at the level of the system) technologized decision-making. In mutating from an artifact to a system, technology carves new boundaries in the distinction between humans and technology. This presents new challenges and opens up an important and novel domain for IS research, the consequences of which must be investigated further.

\section{References}

[1] Alter, S., The Concept of 'It Artifact' Has Outlived Its Usefulness and Should Be Retired Now, Information Systems Journal, 25(1), 2015, 47-60.

[2] Anderson, R.G., Binner, J.M., Hagströmer, B., and Nilsson, B., Liquidity: Systematic Liquidity, Commonality, and High-Frequency Trading, in Handbook of High Frequency Trading, 2015, 197-214.

[3] Angell, I., and Demetis, D., Science's First Mistake: Delusions in Pursuit of Theory, Bloomsbury, London, New York, , 2010.

[4] Backhouse, J., Demetis, D.S., Dyer, B., Canhoto, A., and Nardo, M., Spotlight: New Approaches to Fighting Money-Laundering, http://demetis.wordpress.com, 2005

[5] Bertalanffy, L.V., An Outline of General System Theory, British Journal for the Philosophy of Science, 1(2), 1950, 134-165.

[6] Betancourt, L., Vandenburgh, W., and Harmelink, P., Understanding the 'Flash Crash', The CPA Journal, 2011,

[7] Bloomberg, Sentiment Analysis of Financial News and Social Media, http://www.bloomberglabs.com/datascience/projects/sentiment-analysis-financial-news-socialmedia/, 2015

[8] Boulding, K.E., General Systems Theory - the Skeleton of Science, Management science, 2(3), 1956, 197-208. 
[9] Cftc/Sec, Findings Regarding the Market Events of May 6, 2010: Report of the Staffs of the Cftc and Sec to the Joint Advisory Committee on Emerging Regulatory Issues, http://www.sec.gov/news/studies/2010/marketeventsreport.pdf, 2010

[10] Chaboud, A., Chiquoine, B., Hjalmarsson, E., and Vega, C., Rise of the Machines: Algorithmic Trading in the Foreign Exchange Market, International Finance Discussion Papers, 980 (2009, 1-44.

[11] Cliff, D., and Northrop, L., The Global Financial Markets: An Ultra-Large-Scale Systems Perspective, http://www.gov.uk/government/uploads/system/uploads/att achment data/file/289012/11-1223-dr4-global-financialmarkets-systems-perspective.pdf, 2012

[12] D'amato, A., Can/Should Computers Replace Judges?, Faculty Working Papers, Northwestern University School of Law Scholarly Commons, 1977,

[13] Davies, A., Google's Self-Driving Car Caused Its First Crash, http://www.wired.com/2016/02/googles-selfdriving-car-may-caused-first-crash/, 2016

[14] Demetis, D., and Angell, I., Aml-Related Technologies: A Systemic Risk, Journal of Money Laundering Control, 9(2), 2006, 157-172.

[15] Dhillon, G., and Backhouse, J., Information System Security Management in the New Millennium, Communications of the ACM, 43(7), 2000, 125-128.

[16] Oxford English Dictionary, "Agency", 2016

[17] Hammond, D., The Science of Synthesis : Exploring the Social Implications of General Systems Theory, University Press of Colorado, Boulder, 2003.

[18] Jansen, B., and Spink, A., "An Analysis of Web Documents Retrieved and Viewed", The 4th International Conference on Internet Computing, 2003, 65-69.

[19] Johnston, J., Information Events, Big Data, and the Flash Crash, in Ubiquitous Computing, Complexity and Culture, 2015, 378-392.

[20] Lee, A., and Demetis, D., "Crafting Theory to Satisfy the Requirements of Systems Science", 49th Hawaii International Conference on System Sciences, IEEE, 2016, 5075-5084

[21] Lewis, M., Flash Boys: Cracking the Money Code, Penguin, 2014.

[22] Lhabitant, F.S., and Gregoriou, G.N., High-Frequency Trading: Past, Present, and Future, in Handbook of High Frequency Trading, 2015, 155-166.

[23] Luhmann, N., How Can the Mind Participate in Communication? , in Materialities of Communication, Stanford University Press, 1994, 371-387.

[24] Luhmann, N., Insistence on Systems Theory: Perspectives from Germany, Social Forces, 61(4), 1983, 987-998.

[25] Luhmann, N., Social Systems, Stanford University Press, Stanford, Calif, 1995.

[26] Luhmann, N., System as Difference, Organization, 13(1), 2006, 37-57.

[27] March, S., and Smith, G., Design and Natural Science Research on Information Technology, Decision Support Systems, 15(1995, 251-266.

[28] Markoff, J., Armies of Expensive Lawyers, Replaced by Cheaper Software, http://www.nytimes.com/2011/03/05/science/05legal.html? $\mathrm{r}=0,2011$

[29] Marouli, A., Caloghirou, Y., and Giannini, E., NonPerforming Debt Recovery: Effects of the Greek Crisis, International Journal of Banking, Accounting and Finance, 6(1), 2015, 21-36.

[30] Millar, M., Beating the Recruitment Machines, http://www.bbc.co.uk/news/business-20255387, 2012

[31] Miller, J.G., Living Systems, McGraw-Hill, New York, 1978.

[32] Moosa, I., The Regulation of High-Frequency Trading: A Pragmatic View, Journal of Banking Regulation, 16(1), 2015, 72-88.

[33] Nanex, Analysis of the "Flash Crash" - Part 4, Quote Stuffing,

http://www.nanex.net/20100506/FlashCrashAnalysis Part4 $-1 . h \mathrm{tml}, 2010$

[34] Orlikowski, W., and Iacono, S., Research Commentary: Desperately Seeking the "It" in It Research a Call to Theorizing the It Artifact, Information Systems Research, 12(2), 2001, 121-134.

[35] Postman, N., Technopoly: The Surrender of Culture to Technology, Vintage Books, 1st Vintage Books edn, New York, 1993.

[36] Rapoport, A., Science and the Goals of Man; a Study in Semantic Orientation, Harper, 1st edn, New York, 1950.

[37] Schapiro, M., Testimony Concerning the Severe Market Disruption on May 6, 2010, http://www.sec.gov/news/testimony/2010/ts051110mls.htm , 2010

[38] Silver, M., and Markus, L., Conceptualizing the Sociotechnical Artifact, Systems, Signs \& Actions, 7(2013, 82-89.

[39] Solon, O., How a Book About Flies Came to Be Priced $\$ 24$ Million on Amazon, http://www.wired.com/2011/04/amazon-flies-24-million/, 2011

[40] Sornette, D., and Becke, S.V.D., "Crashes and High Frequency Trading: An Evaluation of Risks Posed by HighSpeed Algorithmic Trading", 2011

[41] Spencer-Brown, G., Laws of Form, George Allen and Unwin Ltd., London, 1969.

[42] Steiner, C., Wall Street's Speed War, http://www.forbes.com/forbes/2010/0927/outfrontnetscape-jim-barksdale-daniel-spivey-wall-street-speedwar.html, 2010

[43] Sutter, J., Amazon Seller Lists Book at \$23,698,655.93 -- Plus Shipping, http://edition.cnn.com/2011/TECH/web/04/25/amazon.pric e.algorithm/, 2011

[44] Van Lier, B., From High Frequency Trading to SelfOrganizing Moral Machines, International Journal of Technoethics, 7(1), 2016, 34-50.

[45] Westbrook, J., High-Frequency Traders Find Microwaves Suit Their Need for Speed, http://www.bloomberg.com/news/articles/2014-07-24/highfrequency-traders-find-microwaves-suit-their-need-forspeed, 2014

[46] Zaydlin, A., Regulating in Response to the "Flash Crash", Economist, 395(8686), 2010 